\title{
Review of Research on Technologies for Language Learning and Teaching
}

\author{
Mengke Yang, Rustam Shadiev \\ School of Education Science, Nanjing Normal University, Nanjing, China \\ Email: Yangmengke2014@163.com,rustamsh@gmail.com
}

How to cite this paper: Yang, M.K. and Shadiev, R. (2019) Review of Research on Technologies for Language Learning and Teaching. Open Journal of Social Sciences, 7, 171-181.

https://doi.org/10.4236/jss.2019.73014

Received: January 28, 2019

Accepted: March 10, 2019

Published: March 13, 2019

\begin{abstract}
We reviewed literature from 2014 to 2018 on foreign/second language learning supported by technologies. We aimed to select empirical studies that show effect of technologies on language learning. In this review study, we describe types of technologies, their potential usage for language learning, advantage and disadvantages, and we also give some examples to understand our findings better. Besides, we compare our results with those obtained from earlier review studies, e.g. we identified several new technologies used in studies between 2014 and 2018 but never used before. We also make several suggestions for future studies in this field. Our results can be useful for teaching and research communities that plan to design technology-enhanced foreign/second language learning and teaching.
\end{abstract}

\section{Keywords}

Technology, Foreign/Second Language Learning, Review

\section{Introduction}

Technology has long been seen as a means of enhancing language learning in many industrial countries. It frees instructors and students from repetitive and boring activities and allows them to focus on the core of language learning. The use of technology, either alone or integrated in the classroom context, was at least as effective as traditional classroom-based learning [1].

The rapid advancement of technologies in the last years has created advantages and opportunities for education [2] that the use of technologies has become nearly ubiquitous for foreign/second language (FL/SL) learning. Some new technologies, such as virtual reality, smart watch and other wearable devices are detected and increasingly available [3]. In addition, some emerging technologies, such as Google glasses and computational thinking, are also maturing and very 
promising to be used in FL/SL learning classrooms. Many studies have shown that technological innovations can promote FL/SL learners' performances, increase their motivation and provide more efficient means for target language input [4] [5].

This study investigated SSCI papers related to technologies used for FL/SL learning from 2014 to 2018. We focused on empirical studies that showing effect of the technology and so as its descriptions of technologies or potential uses. Because of time retrieval conditions, we only focused on relatively new technology. Some technologies such as personal computer and interactive whiteboard are excluded. Because that they are almost totally used by language learners or just out of fashion.

The goal of this study is to give a guide to FL/SL learners, teachers and researchers. For instance, when a teacher wants to use a new technology to assist his instruction, then he can read this paper and see which kind of technology is suitable for his class, what advantages and disadvantages the technology has. Then, we make a comparison between technologies used from 2014 to 2018 and technologies used before 2014. Some new technologies are being used in FL/SL language learning and teaching whereas some old are disappearing.

\section{Method}

This review summarizes researches of technology use in FL learning. Our method followed general recommendations from earlier research [6] [7]. Research articles were searched from Social Sciences Citation Index databases using search terms such as foreign, language, learning, technology and specific name of the technology just like robot, internet of things, wearable devices, and smart watch, etc. This review focuses on empirical researches that showing effectiveness of the technology between 2014 and 2018. So we set criteria and narrowed down the selection of research articles for inclusion. The screening criteria are as follows: first, studies focus on foreign language learning supported by technologies; second, studies that were published between 2014 and 2018; third, studies were written in English; fourth, studies that were published in Social Science Citation Index (SSCI) which are related to educational technology. In order to find the use of unknown technologies in foreign language learning, we also use the retrospective method to collect papers, that is, to further search and verify when the technology that may meet the requirements is found in the selected papers.

In the end, over 50 potentially relevant studies were selected. Because this is work-in-progress, our review work is still ongoing, and only results related to one part of reviewed studies $(n=12)$ was included in this review study.

\section{Result}

We introduce the technologies that applied to FL learning between 2014 and 2018 and divide them into two categories. One is for individual FL/SL learning and the other for collaborative one. There are twelve kinds of technologies that 
we present in this work-in-progress study. We show advantages and disadvantages of the technology and then give a few examples to show how researcher can design their studied and what skills can be improved using these technologies.

\subsection{Technologies That Were Used for Individual Study}

1) Teaching assistant robot

Teaching assistant robot is a kind of robot particularly designed for instruction. Wu, Wang and Chen (2015) [8] designed an experiment to explore the potential for using teaching assistant robots in elementary school. Under the guidance of CLT (communicative language teaching), TPR (Total physical response) and teaching storytelling, they designed a teaching assistant robot on their own to support their teaching content such as 26 English letters, self-introduction and body parts, conversation, and storytelling. The main skill is communication. So the teaching assistant robot had the capability to exhibit various forms of communication and interaction with the student learners, such as facial expression, gestures, and motions on wheels.

In their study, they found that teaching assistant robots did yield a better learning outcome. As a novel technology, teaching assistant robots made students fascinated and remained closely focused and engaged. It captured students' attention quickly and held their attention during the whole sessions and also motivated them to study English. Besides, the physical appearance was found to be an important factor. Human-like or cartoon-like external appearances are more acceptable. A familiar, interesting look will be effective to those who were often afraid to speak up or answer questions in English in front of their classmates. However, there is still something wrong of voice recognition in a noisy environment.

2) Corpus

Claims about corpus have been going on for years. But the evidence regarding the effectiveness of corpus in FL learning comes from qualitative studies or case studies before 2014. Daskalovska (2015) [9] designed an experiment to investigate the effectiveness of corpus-based activities for learning verb-adverb collocations compared to traditional activities usually found in course books. The test results show that the participants who learned with the help of corpus gained more knowledge of verb-adverb collocations than the control group. Corpus was proofed to be a powerful tool in the hands of English as a foreign language (EFL) student.

With the help of corpus, students can get more varied and detailed information about the words and collocations. When students search for words, it will show a large number of authentic examples of the use of these words in various contexts so that students can see how they are used, in what contexts they are used, and also see the surrounding words or structures. In this way, students will spend some time analyzing and interpreting the information, which means there is depth of information processing takes place. So they can learn better and re- 
member new knowledge longer.

3) $3 \mathrm{D}$ virtual worlds (Chabot and time machine)

3D virtual worlds are used to construct learning situations that simulate realistic scenarios. It is distinguished from other types of media through immersion and presence. FL learners can take advantage of hypothetically real simulations in graphically rich and dynamic environments (Dalgarno \& Lee, 2010) [10] and immerse themselves to practice their target language skills in contexts.

Wang, Petrina and Feng (2015) [11] developed an immersive English learning environment with two key learning artifacts, Chabot and time machine. These two learning artifacts mainly aimed at facilitating learning verbs. Language learners were expected to learn how to distinguish linking verbs, action verbs and participles. Besides, they are also expected to construct sentences using present, past and future tenses as well as active and passive voices. The experimental results indicate that the Chabot and time machine increase the learners' sense of immersion and presence which means $3 \mathrm{D}$ virtual worlds performed well in immersion as well as in FL learning.

4) Automated written corrective feedback

To some extent, automated written corrective feedback (AWCF) is a substitute of teacher-provided written corrective feedback (WCF). Li, Link, and Hegelheimer (2015) [12] said that AWCF is useful to lighten teachers' feedback burden. For example, AWCF can deal with problems in sentence-level grammar so that teachers can focus more on higher-level concerns such as content and discourse. It is useful for students too because they can use AWCF freely. Then, revise and proofread their own work on time.

Ranalli (2018) [13] used an AWCF-based error-correction task in his research which related to EFL writing. In his study, the explicitness and accuracy of the feedback was controlled and so as students' response type. Students could only modify the words according to AWCF, not delete directly. Results showed that explicitness is an important determining factor for L2 students to correct written errors. For example, generic feedback required more mental-effort expenditure than specific feedback but less clear and helpful. But the need to evaluate accuracy didn't call for unique mental effort.

Of course, AWCF is a valuable technology. For teachers, it can lighten their feedback burden. And for students, it can improve the quality of writing across drafts of text. But there are still some limitations of AWCF such as its one-size-fits-all nature. AWCF takes little account of individual differences because its error types are determined more by technological capacities than pedagogical considerations.

5) Online game-based platform

Online game-based platform is implemented like a game-based learning management system (LMS) platform that provides the functionality for game, communication, storage, and receiving data online. Game makes this kind of 
technology more fascinating and engaging.

Usai, Neil and Newman (2017) [14] describe an online game-based platform named LANGA for L2 learning and research. And present a demonstrative proof-of-concept study using the platform. The result of their study showed that with the help of platform, participants were able to learn a large proportion of the new words, and retained the novel words longer. They also claimed that the online game-based platform is effective and engaging for the consumer.

6) Internet of things and wearable technologies

The Internet of things (IoT) is a technology based on the Internet that extends its terminal to any objects so as to carry out information exchange and communication. Wearable devices are portable devices worn directly on the body such as glasses. It can achieve powerful functions through software support, data interaction and cloud interaction.

Nowadays, more and more educators believe that situating foreign language learners in authentic environment has become an important issue especially for young children who need to learn a new language by concrete vocabulary [15]. IoT and wearable technologies provide instructors with tools to create and manage scenarios including everyday objects and friendly interfaces so that young learners can handle and see them directly.

In a research related to task-based foreign language learning for young children, Elena and other colleagues [16] used Internet of things (IoT) and wearable technologies to create realistic task-based language learning scenarios. As they found, the use of these technologies is beneficial. In class, Internet of things and wearable technologies can free instructors of keeping records performed by each student during the tasks. Instead, these technologies allow instructors focus their efforts on creating a friendly environment and encouraging students to participate in learning process more actively.

7) Virtual reality

Virtual reality (VR) is a computer-generated interactive experience which takes place within a simulated environment. That usually simulates parts of our world or completely an imaginary world using high-performance computers and sensory equipment. These wearable devices visually isolate users from the physical real-world surroundings.

The emergence of VR technology enables FL learners to have cultural or language immersion without putting their physical bodies in the target language environment, thus creating a feeling of being there. Virtual environments create an avatar for each learner, and then let it walk through various locations and interact with other avatars in the target language from different cultures. Learning cultural knowledge in the target language from people representing it (host of the target culture/language) is critical to FL learners. Cultural immersion allows FL learners to learn target language meaningful and effective by observing, participating, and engaging with language and culture.

Shih (2015) [17] did a research that combined a qualitative case study with a 
time-series design to investigate the impact of virtual context on culture learning. The results show that all of the participants involved in the study benefitted from their cultural immersion in the virtual environment. That means VR could help enhance learner cultural knowledge and language learning. Besides, VR allowed widely access (even over distance) learning resources, highly engage in learning experiences so that it could motivate learners to attempt just-in-time and trial-and-error FL/SL learning without risk.

8) Augmented reality

Augmented reality (AR) is a kind of technology that enhances the sense of reality by integrating digital information and real environments. Many people confuse VR with AR. The difference of these two technologies is that VR completely replaces real world environment with simulated environment, while AR combines or supplements real world objects with virtual objects or digital information. In an AR supported environment, the objects exist in real-world are "augmented" by computer-generated perceptual information. This process involves multiple sensory organs such as visual, auditory, haptic, somatosensory, and olfactory like VR supported activities.

Hsu (2017) [18] has developed two AR educational game systems for third graders (no-English native speaker) to learn English vocabulary in free and situated surroundings. They found that AR can provide such as ubiquitous and situated learning and learners' senses of presence and immediacy. Besides, some skills such as information management, problem solving or reflection can also be potentially motivate and strengthen. Ho, Hsieh, Sun and Chen (2017) [19] used AR features to develop a ubiquitous learning instruction system in order to improve the performance of EFL learning with authentic situations. Results of their study showed that using AR can positively improve FL learners' learning performance.

9) Smart watch

A smartwatch is a general-purpose, internet-connected computer worn on wrist with multiple sensors that continuously measure and display different information for the user [20].

The most prominent function of the smartwatch is to monitor the user's movement status in real time, such as recording the number of steps taken every day. Therefore, smart watches are very suitable for supporting language learning in sports. In this way, learners can not only learn language knowledge but also exercise their bodies.

Shadiev, Hwang and Liu (2018) [21] carried out a single subject experiment to research the affordances of smart watches for EFL learning and healthy and enjoyable living. In their study, they designed an EFL learning activity using smart watches to combine EFL learning with physical exercise. The learners participated in learning activity using smart watches during the first week, and without smart watch support during the second week. The result showed that the when learners used smart watches, they performed better. The questionnaire and interview results showed that the smart watches were easy to use and useful for 
EFL learning, health and positive emotions. Their study also suggested the advantages of smart watch. The first one is wireless connectivity. It allows students seamlessly access to learning information and interact with teachers and peers. The second is creating content using the multimedia tools. The third is receiving feedback or notifications timely. But the screen size of smart watch is too small and, therefore, may be too inconvenient when inputting learning content into smartwatch, e.g. typing.

\subsection{Technologies That Call for Collaboration}

1) Mobile immersion

Immersion is known as an acclaimed learning approach for second language (L2) acquisition. But putting learners into a real environment such as a foreign country or international school is too costly and not available for most learners. And now people can use their mobile devices to create a mobile immersion that hope to replace essence of immersion.

Lai (2016) [22] created a mobile immersion environment on a smartphone using a mobile instant messenger, "WhatsApp ${ }^{\text {rw }}$. The participants (non-native English speaker) of experimental group were put into a chat group and asked to use English in their daily lives to chat with their teammates using written text. The aim of their study was to explore whether mobile immersion significantly improve the learning of high-frequency English verbs. Their results did not show a significant difference between vocabulary gains of experimental group and control group but showed significant correlation between individual vocabulary gain and chat frequency. That means, although mobile immersion is cheaper and more convenient than traditional immersion but its effect is greatly influenced by the user's mentality.

2) Wikis

As a web-based social networking platform, wikis can be adopted into an instrumental tool for teachers to help students acquire writing skills [23]. Because of its free editing and review structure [24], wikis can provide multiple functions to teachers and students, such as communication, sharing materials, editing files and collaborating on documents within a shared space [25]. But some learners are also worried about the uneven distribution of work among participants [26].

Wang (2015) [27] examined the production and interaction of English for specific purposes learners in a wiki learning environment. Students of experimental group were asked to draft, peer-edit, and revise two written assignments through the wiki while students of control group were asked to work in groups to perform the same tasks in a non-wiki environment. According to the pre-test and post-test, both groups have significant improvements in business writing but the experimental group performed better. Result of questionnaire also suggested that wikis promote students' interest in language learning and boost the development of their writing competencies.

3) WeChat 
WeChat is one of the most popular Social networking platforms in Chinese-speaking communities. For non-Chinese native speakers, WeChat can be a powerful tool for Chinese as FL/SL learning. It provides variety of functions such as instant and direct communication with WeChat friends, building their own "Moments" and payment. Besides, people can also use it to support online shopping, bank transactions, and arranging hospital appointments. These kinds of functions are usually implemented by programs embedded in WeChat.

WeChat has existed for a long time, and it has been constantly improving and its functions are more abundant than before, allowing many types of information such as text, emoji, pictures, audio and video. Although WeChat has rich functions, it is not the mainstream way for social communication in other languages. However, since WeCHat is popular among Chinese speaking population, it can be used for Chinese as FL/SL learning.

Jin (2018) [4] used a qualitative research method to investigate what affordances of WeChat for language development are. Jin (2018) identified four affordances. He found that WeChat is a casual space with easy access to native speakers of Chinese. It provided authentic meaning-focused communication with native speakers of Chinese, linguistic resources, multi-literacies and space for new identity creation. The results show that due to unique communication proficiency, personal preference and norms on WeChat, each affordance was verified differently for different Chinese language learners.

\section{Discussion and Conclusion}

In our review, we focused on the usage and effectiveness of technologies for FL/SL learning. We gave research examples, showed how to use it and discussed advantages and disadvantages of technologies one by one. All of the twelve kinds of technologies we discussed in this review were showed that they are useful for FL/SL learning. When teachers want to use tools to enhance FL/SL learning performance, our results can be useful to guide their learning activity design. And from the research example, they also can have an idea of taking advantage of the technology and avoiding its disadvantages.

There is a limitation of this review study that needs to be acknowledged and addressed in the future. That is, our review was limited by the search terms. We used various search terms in order to present more kinds of new technologies on reviewed studies. Perhaps, involving some experts on educational technologies for language learning can be useful to extend our search range.

\subsection{Comparison of New and Old Technologies}

We make a comparison between technologies in this review and technologies used before 2014. Table 1 shows what new technologies have emerged in foreign language learning, what are old and disappearing and what are still in use. We should know that the technologies which are still in use is not same as the old one but become more powerful and have more digital affordances. 
Table 1. Comparison with new technologies, old technologies and technologies that are still in use.

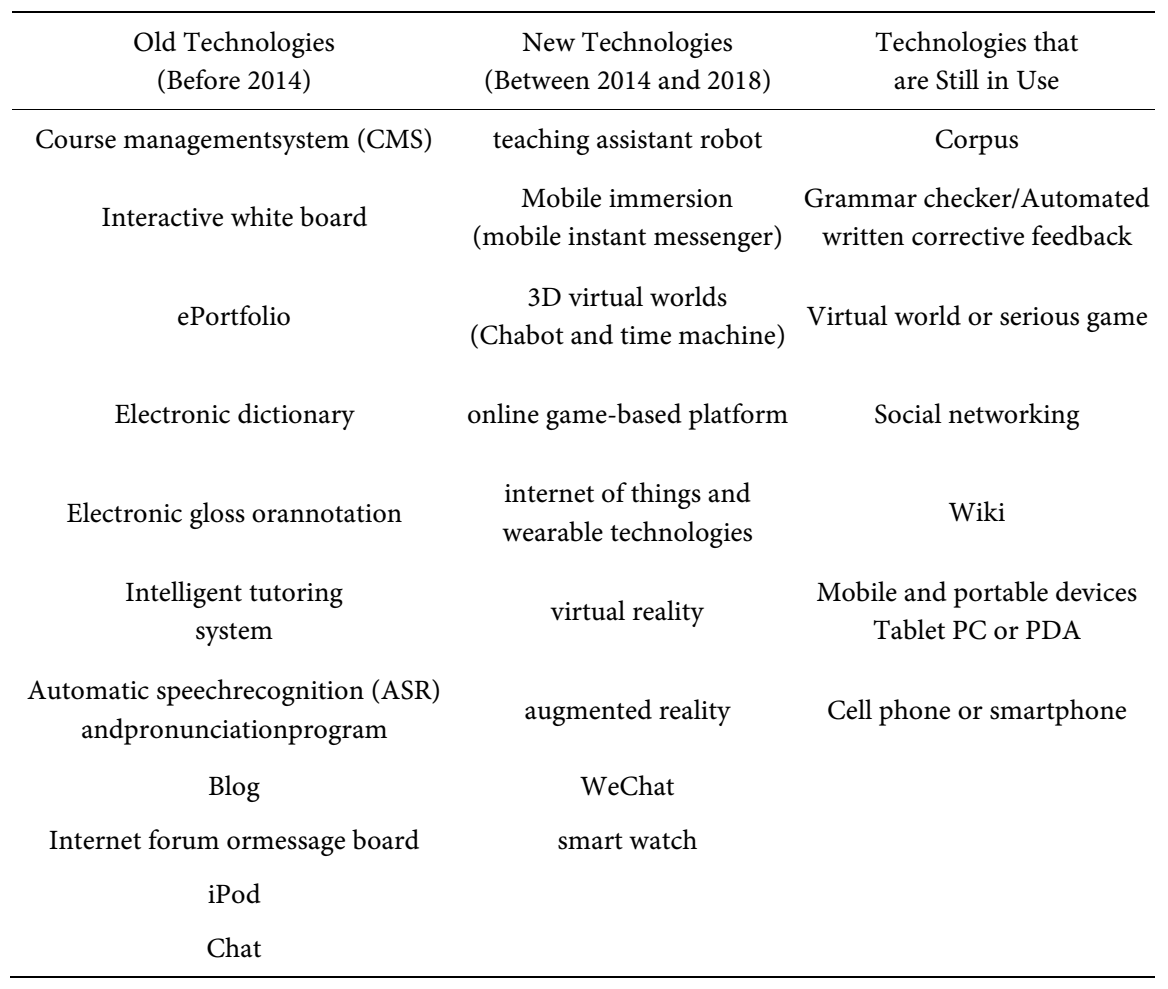

\subsection{The Promising Technology for Language Learning and Research}

In addition to all the new technologies mentioned in this article that have been applied to foreign language learning and the widely used technologies that we are already familiar with, such as computers, tape recorders and televisions, there are still some very novel technologies that have not been applied to foreign language learning. Some of such new technologies are Google glass, computational thinking, air three-dimensional imaging technology, edge computing, natural language processing, etc.

We suggest that these technologies are still in the process of maturation and not many scholars have applied them to FL/SL learning yet. However, it cannot be denied that these technologies are likely to become an important force in promoting FL/SL learning in the future [7].

\section{Annotation}

The literature with ${ }^{*}$ belongs to the scope of papers which are reviewed.

\section{Conflicts of Interest}

The authors declare no conflicts of interest regarding the publication of this paper.

\section{References}

[1] Maja, G., Chapelle, C.A. and Shelley, M.C. (2013) A Meta-Analysis of Effectiveness 
Studies on Computer Technology-Supported Language Learning. ReCALL, 25, 34 .

[2] Reynolds, B.L. and Anderson, T.A.F. (2015) Extra-Dimensional In-Class Communications: Action Research Exploring Text Chat Support of Face-to-Face Writing. Computers and Composition, 35, 52-64. https://doi.org/10.1016/j.compcom.2014.12.002

[3] Shadiev, R., Sun, A. and Huang, Y.M. (In Press) A Study of the Facilitation of Cross-Cultural Understanding and Intercultural Sensitivity Using Speech-Enabled Language Translation Technology. British Journal of Educational Technology. https://doi.org/10.1111/bjet.12648

[4] Jin, L. (2018) Digital Affordances on WeChat: Learning Chinese as a Second Language. Computer Assisted Language Learning, 31, 1-26. https://doi.org/10.1080/09588221.2017.1376687

[5] Shadiev, R. and Huang, Y.M. (In Press) Investigating Student Attention, Meditation, Cognitive Load, and Satisfaction during Lectures in a Foreign Language Supported by Speech-Enabled Language Translation. Computer Assisted Language Learning.

[6] Shadiev, R., Hwang, W.Y. and Huang, Y.M (2017) Review of Research on Mobile Language Learning in Authentic Environments. Computer Assisted Language Learning, 30, 284-303. https://doi.org/10.1080/09588221.2017.1308383

[7] Shadiev, R., Hwang, W.Y., Chen, N.S. and Huang, Y.M. (2014) Review of Speech-to-Text Recognition Technology for Enhancing Learning. Educational Technology \& Society, 17, 65-84.

[8] Wu, W.C.V., Wang, R.J. and Chen, N.S. (2015) Instructional Design Using an In-House Built Teaching Assistant Robot to Enhance Elementary School English-as-a-Foreign-Language Learning. Interactive Learning Environments, 23, 696-714. https://doi.org/10.1080/10494820.2013.792844

[9] Daskalovska, N. (2015) Corpus-Based versus Traditional Learning of Collocations. Corpus-Based versus Traditional Learning of Collocations.

[10] Dalgarno, B. and Lee, M. (2010) What Are the Learning Affordances of 3_D Virtual Environments? British Journal of Educational Technology, 41, 10-32. https://doi.org/10.1111/j.1467-8535.2009.01038.x

[11] Wang, Y.F., Petrina, S. and Feng, F. (2015) Village-Virtual Immersive Language Learning and Gaming Environment: Immersion and Presence. British Journal of Educational Technology, 48, n/a-n/a.

[12] Li, J., Link, S. and Hegelheimer, V. (2015) Rethinking the Role of Automated Writing Evaluation (AWE) Feedback in ESL Writing Instruction. Journal of Second Language Writing, 27, 1-18. https://doi.org/10.1016/j.jslw.2014.10.004

[13] Ranalli, J. (2018) Automated Written Corrective Feedback: How Well Can Students Make Use of It? Computer Assisted Language Learning, 1-22.

[14] Usai, F., O’Neil, K. and Newman, A.J. (2017) Design and Empirical Validation of Effectiveness of Langa, an Online Game-Based Platform for Second Language Learning. IEEE Transactions on Learning Technologies, 1-1.

[15] Antle, A.N. and Wise, A.F. (2013) Getting Down to Details: Using Theories of Cognition and Learning to Inform Tangible User Interface Design. Interacting with Computers, 25, 1-20. https://doi.org/10.1093/iwc/iws007

[16] Elena, D.L.G., Camacho, V., Orozco-Barbosa, L., Lujan, V.B., Penichet, V.M.R. and Lozano, M. (2016) Introducing IOT and Wearable Technologies into Task-Based Language Learning for Young Children. IEEE Transactions on Learning Technolo- 
gies, 9, 366-378. https://doi.org/10.1109/TLT.2016.2557333

[17] Shih, Y.C. (2015) A Virtual Walk through London: Culture Learning through a Cultural Immersion Experience. Computer Assisted Language Learning, 28, 407-428. https://doi.org/10.1080/09588221.2013.851703

[18] Hsu, T.-C. (2017) Learning English with Augmented Reality: Do Learning Styles matter? Computers \& Education, 106, 137-149. https://doi.org/10.1016/j.compedu.2016.12.007

[19] Ho, S.-C., Hsieh, S.-W., Sun, P.-C. and Chen, C.-M. (2017) To Activate English Learning: Listen and Speak in Real Life Context with an AR Featured U-Learning System. Educational Technology \& Society, 20, 176-187.

[20] Reeder, B. and David, A. (2016) Health at Hand: A Systematic Review of Smart Watch Uses for Health and Wellness. Journal of Biomedical Informatics, 63, 269-276. https://doi.org/10.1016/j.jbi.2016.09.001

[21] Shadiev, R., Hwang, W.-Y. and Liu, T.-Y. (2018) A Study of the Use of Wearable Devices for Healthy and Enjoyable English as a Foreign Language Learning in Authentic Contexts. Educational Technology \& Society, 21, 217-231.

[22] Lai, A. (2016) Mobile Immersion: An Experiment Using Mobile Instant Messenger to Support Second-Language Learning. Interactive Learning Environments, 24, 277-290. https://doi.org/10.1080/10494820.2015.1113706

[23] Kessler, G. and Bikowski, D. (2010) Developing Collaborative Autonomous Learning Abilities in Computer Meditated Language Learning: Attention to Meaning among Students in Wiki Space. Computer Assisted Language Learning, 23, 41-58. https://doi.org/10.1080/09588220903467335

[24] Parker, K.R. and Chao, J.T. (2007) Wiki as a Teaching Tool. Interdisciplinary Journal of Knowledge and Learning Objects, 3, 57-72. https://doi.org/10.28945/3131

[25] Boulous, M., Maramba, I. and Wheeler, S. (2006) Wikis, Blogs and Podcasts: A New Generation of Web-Based Tools for Virtual Collaborative Clinical Practice and Education. BMC Medical Education, 6, 41. https://doi.org/10.1186/1472-6920-6-41

[26] Alyousef, H. and Picard, M. (2011) Cooperative or collaborative Literacy Practices: Mapping Metadiscourse in a Business Students' Wiki Group Project. Australasian Journal of Educational Technology, 27, 463-480. https://doi.org/10.14742/ajet.955

[27] Wang, Y.C. (2015) Promoting Collaborative Writing through Wikis: A New Approach for Advancing Innovative and Active Learning in an ESP Context. Computer Assisted Language Learning, 28, 499-512. https://doi.org/10.1080/09588221.2014.881386 